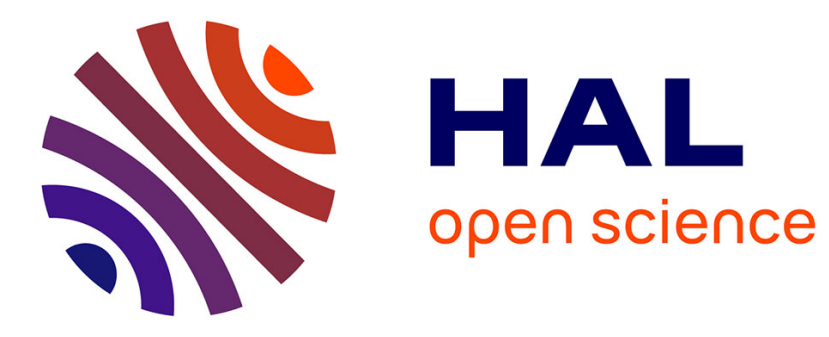

\title{
A submerged freeze microgripper for micromanipulations.
}

Beatriz Lopez-Walle, Michaël Gauthier, Nicolas Chaillet

\section{To cite this version:}

Beatriz Lopez-Walle, Michaël Gauthier, Nicolas Chaillet. A submerged freeze microgripper for micromanipulations.. IEEE International Conference on Robotics and Automation. ICRA'2007., Apr 2007, Rome, Italy. pp.826-831. hal-00162325

\section{HAL Id: hal-00162325 https://hal.science/hal-00162325}

Submitted on 13 Jul 2007

HAL is a multi-disciplinary open access archive for the deposit and dissemination of scientific research documents, whether they are published or not. The documents may come from teaching and research institutions in France or abroad, or from public or private research centers.
L'archive ouverte pluridisciplinaire HAL, est destinée au dépôt et à la diffusion de documents scientifiques de niveau recherche, publiés ou non, émanant des établissements d'enseignement et de recherche français ou étrangers, des laboratoires publics ou privés. 


\title{
A Submerged Freeze Microgripper for Micromanipulations
}

\author{
Beatriz López Walle, Michaël Gauthier, and Nicolas Chaillet
}

\begin{abstract}
Efficient, reliable and flexible handling is still very challenging in micromanipulation and micro-assembly. In this paper, we propose an original thermally actuated gripper based on the use of ice to manipulate submerged artificial micro-objects sized under $100 \mu \mathrm{m}$. Manipulating in liquid surroundings can indeed be more interesting than in dry conditions. A comparative analysis on the impact of dry and liquid media on surface forces, contact forces and hydrodynamic forces shortly given first shows it. Concerning the use of ice for micromanipulation, cryogenic grippers are a flexible solution. Nevertheless, as they currently work in air, water must be provided by an external device and capillary force occurs during the release. Our submerged freeze microgripper takes advantages of the aqueous surroundings for the handling process as explained. The thermal principle, based on the Peltier effect, the characteristics of the microgripper prototype and the first micromanipulation tests are also presented. To control and optimize the heat exchanges in the developed gripper, a static thermal model using electrical analogy has been developed and validated for the Peltier elements and a 3D heat sink of the gripper. Further work will focus on the dynamic equivalent electric model.
\end{abstract}

\section{INTRODUCTION}

The increasing complexity of the microsystems requires different and new micromanipulation processes and micro-assembly technologies and methods [1]. A main approach in this domain is the robotic assembly which seems relevant for a small production batch [2], [3]. Current manipulation strategies do not allow sufficient reliability and flexibility for micro-objects of which typical dimension is below $100 \mu \mathrm{m}$. When the dimension of the objects falls under this size, surface forces permanently influences the interactions between the object and its surroundings [4]. Their impact perturbs especially the release of the micro-object.

Surface forces, contact forces and hydrodynamic forces strongly depend on environmental parameters [5]. An original way to reduce adhesion perturbations consists in performing micromanipulation tasks being totally submerged in a liquid medium.

Van der Waals forces are proportional to Hamaker constant, which reduces in liquid medium [6]. In liquid, double layer, solvation and steric forces have also to be considered. These forces, modelled by the XDLVO theory

This work is supported by the PRONOMIA project granted by the ANR French National Research Agency

Authors are with Lab. d'Automatique de Besançon, UMR CNRS 6596 - ENSMM - UFC, 24 rue Savary, 25000 Besançon, France blopez@ens $2 \mathrm{~m} . \mathrm{fr}$
[7], [8], are normally repulsive and consequently reduce the effect of attractive van der Waals forces.

Electrostatic forces are also greatly reduced in water: the dielectric constant is higher and the electric conductivity is better in water than in air.

The capillary force between a gripper and an object often induced in air by ambient humidity totally disappears in a liquid medium.

The pull-off force, which represents the necessary force to break the contact between two objects, is proportional to the surface energy between these two objects, and decreases when objects are submerged [6].

Finally hydrodynamic force limits the maximal velocity of the micro-object, decreasing their loss rate. However, the maximum velocity of the microgripper endeffectors has also to be limited to avoid disturbances.

[9], [10] gives a detailed comparative analysis of these forces in liquid and dry surroundings. Considering thus the reduction of surface and contact forces, and the limitation of micro-objects loss caused by the hydrodynamic forces, liquid medium is a promising approach to perform micro-assembly.

Though the adhesion effects are reduced, they do not disappear and could always perturb release phases. Consequently, original micromanipulation strategies have to be proposed to guarantee reliable and repeatable micromanipulation tasks. In this paper, we propose a new freeze microgripper to manipulate micro-objects in a submerged medium.

Existing cryogenic grippers work in air, as presented in [11]-[13], using adhesive properties of ice for handling. In this case, water must be provided by an external device and capillary force can appear during the release because water does not evaporate completely. They have to combine thus additive release strategies as the use of adhesive surface to place and detach manipulated objects. The gripped objects are typically, bigger than $200 \mu \mathrm{m}$. The miniaturization of these grippers is limited by the release difficulties.

In the submerged freeze gripper proposed in this paper, capillary force does not influence the handling procedure. Consequently, it can be extended to smaller objects below $100 \mu \mathrm{m}$. Main applications are micro-assembly of optical, mechanical, electrical and even biological microcomponents. Cryogenic grippers do not cause damages to object surfaces, provide high holding forces without introducing additional stress, and their handling abilities are almost independent of objects shape and material 
properties (low thermal conductive objects are nevertheless recommended).

The proposed handling strategy for submerged micromanipulations is presented in Fig. 1. In the first step, the gripper approaches to the object without touching it. In the second step, an ice microdrop is generated covering just a small part of the object or completely. After this, the object can be picked and positioned. Finally, in the last step, the ice droplet is thawed and the object is liberated without adhesion perturbations. Inserting micro-objects into the water tank, recuperating them, and drying them must be analysed yet.

The thermal validation has been previously modelled with the finite elements software (FEM) COMSOL Multiphysics 3.2. Results are detailed in [14].

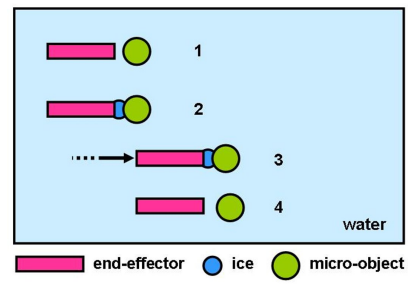

Fig. 1. Handling strategy: (1) the micro-gripper approaches, (2) an ice droplet is generated and catch the object, (3) the object is manipulated, (4) the ice thaws and the object is liberated

This paper is structured in two main sections. Section II describes the principle of the submerged freeze gripper, the characteristics of the experimental device, and its first tests. Section III deals with the thermal modelling of the device using an equivalent electrical model.

\section{EXPERIMENTAL DEVICE}

A first prototype of the submerged freeze gripper has been developed. The whole system principle based on the Peltier effect, its physical characteristics and a manipulation test are described in this section.

\section{A. Principle of the Submerged Freeze Gripper}

The basic idea is to grip a micro-object using an ice droplet formed and controlled in an aqueous medium, then to manipulate the micro-object, and finally to release it through a controlled ice thawing. To carry out this sequence a specific microgripper was developed. The necessary cooling and heating energy to actuate the microgripper is obtained by two thermoelectrics Peltier modules. Classically a Peltier module is the association of bismuth-telluride $\mathrm{p}$ and n-doped semiconductors connected electrically in series and thermally in parallel. A Peltier module gives an electrical current-proportional generation or absorption of heat when current flows through it. The direction of the heat flow depends on the direction of the current. The difference of temperatures caused by the heat transfer imposes two faces: a cold face, and a hot face. The hot face is associated to a heat sink which dissipates the heat flux.
The submerged freeze system is composed of two Peltier modules stages and a forced convection system, as illustrated in Fig. 2. The first module, named MicroPelt $(\mu P)$, provides the cooling energy to generate the ice droplet on the end-effector, which is directly attached to its cold side. The freezing process increases the temperature of the MicroPelt's hot face. The convection is thus so important than the whole system (liquid, gripper and Peltier micromodule) could warm up. That is why a second Peltier module, called MiniPeltier $(m P)$, is also used to actively decrease the temperature on the MicroPelt's heat sink. To optimize the performance of the MiniPeltier, the temperature of its hot face must be constant. This temperature is so maintained at the ambient temperature by forced convection using a liquid cooling system. The liquid cooling system can not be used directly on the MicroPelt's hot face because of its small size $(720 \mu \mathrm{m} \times 1420 \mu \mathrm{m})$. In addition, its maximal cooling capacity is not enough to freezing the end-effector from ambient temperature.

The MiniPeltier and the cooling liquid system stay in air to dissipate heat outside water. The end-effector and the MicroPelt are completely submerged and electrically insulated.

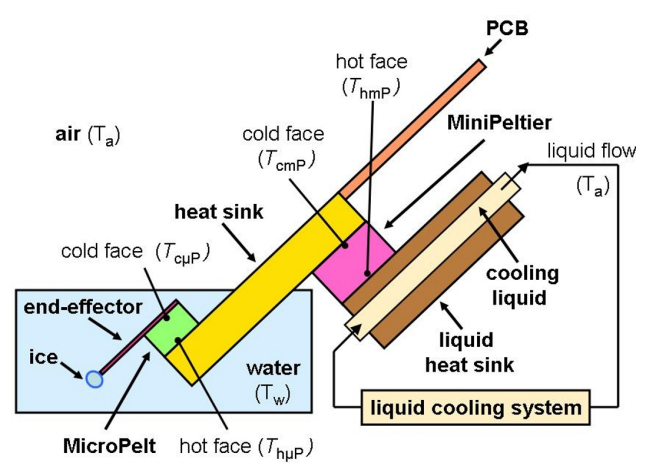

Fig. 2. Submerged freeze microgripper principle

Experimentations verify that the temperature on the MiniPeltier's hot face $\left(T_{h m P}\right)$ remains almost constant when its electrical current $\left(i_{m P}\right)$ ranges from $0 \mathrm{~A}$ to its maximal value $1.1 \mathrm{~A}$. The difference between the ambient temperature $\left(T_{a}\right)$ and the hot face temperature is lower than $2^{\circ} \mathrm{C}$, as appreciate in Table I. Ambient temperature in this test was $21.3^{\circ} \mathrm{C}$.

TABLE I

Temperature at the Hot Face of the MiniPeltier

\begin{tabular}{|l|c|c|c|c|}
\hline Current $i_{m P}(\mathbf{A})$ & 0 & 0.39 & 0.79 & 1.10 \\
\hline Temperature $T_{h m P}\left({ }^{\circ} \mathbf{C}\right)$ & 21.3 & 21.5 & 22.0 & 22.4 \\
\hline
\end{tabular}

\section{B. Physical and Technical Characteristics}

The first prototype of the submerged freeze gripper (without the end-effector) is shown in Fig. 3. 


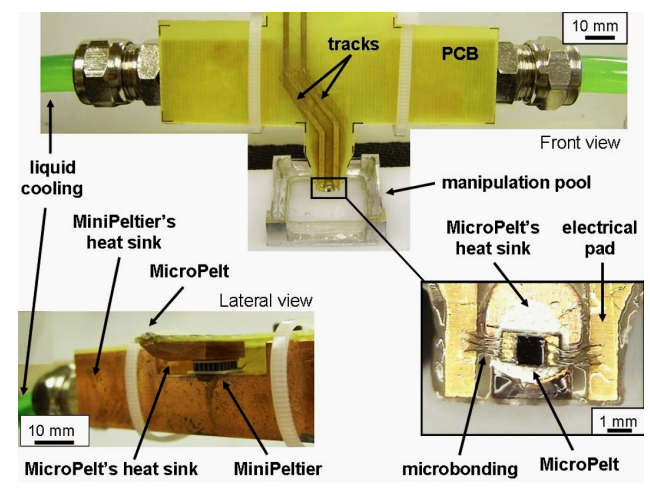

Fig. 3. Experimental freeze gripper

The dimensions of the MicroPelt (from Infineon Technologies AG) are: $720 \times 720 \times 428 \mu \mathrm{m}^{3}$. Its heat sink is fixed, on one side, to the MicroPelt's hot face, and on the other side, to the MiniPeltier's cold face. The dimensions of the MiniPeltier (Melcor FC0.6-18-05) are: $6.2 \times 6.2 \times$ $2.4 \mathrm{~mm}^{3}$. Its hot face is fixed to the copper liquid heat sink of the cooling liquid system.

A specific PCB has been fabricated to make the electrical connections of the Peltier modules. Because of the very small dimensions at stake, microbonding technology were used for its connection to the MicroPelt.

\section{First Experimentations}

First experimentations using the prototype described above in water surroundings were performed. The objectives were to validate the good working of this new system and its reliability. For these tests, the end-effector was not included. The active part was then the MicroPelt's cold face; manipulated objects corresponds thus to its dimensions. However, the handling principle to manipulate micro-objects under $100 \mu \mathrm{m}$ is verified in [14].

Fig. 4 describes the tele-manipulation of a silicon object whose dimensions are: $600 \times 600 \times 100 \mu \mathrm{m}^{3}$. The micromanipulation has been performed in $30 \mathrm{~s}$. This time can be optimize automating micromanipulations. A precooling phase is necessary to decrease the temperature of the MicroPelt's heat sink. During this phase, only the current in the MiniPeltier $\left(i_{m P}\right)$ is applied and set constant at $0.9 \mathrm{~A}$ (Fig. 4a). When the temperature is about $0.5{ }^{\circ} \mathrm{C}$ (this temperature is sufficiently close to 0 ${ }^{o} \mathrm{C}$ but it prevents the heat sink to freeze), the MicroPelt is approached to the micro-object and its current $\left(i_{\mu P}\right)$ is turned on at $0.5 \mathrm{~A}$. The cooling energy generates the ice droplet $(4 \mu \mathrm{l})$ which involves part of the object in $3 \mathrm{~s}$ (Fig. 4b). The freeze gripper can thus displace it towards a new position (Fig. 4c). To release it, the MicroPelt's current is inverted at $-0.3 \mathrm{~A}$. The ice droplet thaws in $7 \mathrm{~s}$ and melts with the medium, liberating the microobject without adhesion perturbations (Fig. 4d). In fact, contrary to the cryogenics gripper in air, capillary forces do not perturb the release because the object and the MicroPelt are submerged.

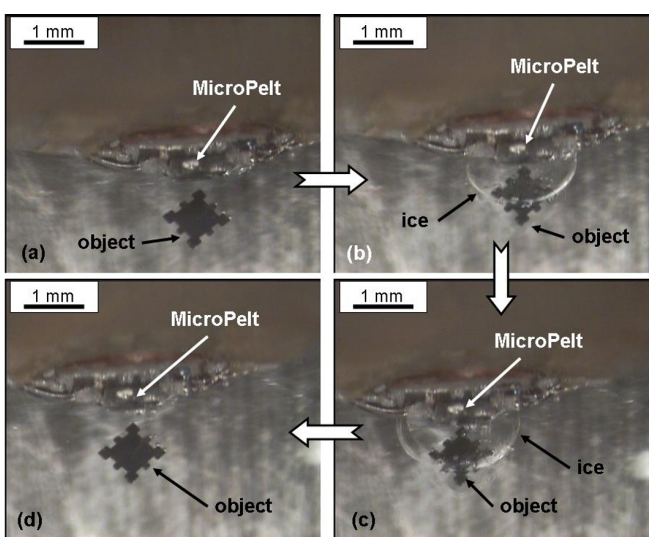

Fig. 4. Micromanipulation of a $600 \times 600 \times 100 \mu \mathrm{m}^{3}$ silicon object with the submerged freeze gripper

The same experiment was successfully repeated several times. The submerged freeze principle seems a promising approach to manipulate micro-objects. Further manipulations will be dedicated to objects sized under $100 \mu \mathrm{m}$.

To maximize the cooling energy to the end-effector and optimize the cycle time, it is necessary to control the cold temperature of both Peltier elements, the MicroPelt and the MiniPeltier, via their electrical currents. The definition of the control strategy requires a model of the thermal exchanges in the whole system. Next section deals with the static modelling of both Peltier elements and a heat sink by electrical analogy.

\section{ELECTRICAL MODEL}

Thermal modelling and control is a crucial part of the design of the submerged freeze system. An equivalent electrical model, so-called thermal network, represents a relatively simple, but powerful tool for simulating the real system [15]. It notably allows to easily connect several thermal subsystems together. Classical electrical models correspond in general to planar thermal systems. Consequently, they can not be applied to our 3D device. For all these reasons, and considering the complexity of heat transfers on the submerged freeze gripper, we propose to build up its proper thermal network. This section reminds first the bases of the electrical analogy for thermal systems; then, the Peltier module's electrical modelling is presented; and finally, we propose the model of a 3D heat sink. This paper focus on the description of the steady state models of our microgripper.

\section{A. Bases of Electrical Analogy for 1D Thermal Problem}

An equivalent electrical model usually facilitates and simplifies the analysis of a thermal problem. Used relationship between thermal and electrical systems are summarized in table II.

Fig. 5 illustrates the heat transfer in a multilayer wall and its equivalent electrical circuit. It corresponds to the 
TABLE II

Thermal and Electrical Analogy

\begin{tabular}{|l|l|}
\hline Thermal system & Equivalent electrical system \\
\hline Heat flux $Q$ & Current $i$ \\
\hline Temperature difference $\Delta T$ & Voltage difference $\Delta V$ \\
\hline Thermal resistance $R_{t h}$ & Resistance $R$ \\
\hline
\end{tabular}

typical example for the application of this concept. In this case, heat convection takes place between the lateral surfaces and the external fluids, and heat conduction takes place in the layers. Convection thermal resistance $R_{\text {conv }}$ is a function of the convective heat transfer coefficient $h$ and the lateral surface $S$ of the wall:

$$
R_{\text {conv }}=1 / h S,
$$

while conduction thermal resistance $R_{\text {cond }}$ is obtained according to the thickness $e$ of the layer, its thermal conductivity $k$, and the lateral surface $S$ :

$$
R_{\text {cond }}=e / k S .
$$

Then, the steady state model of this multilayer wall can be obtained in connecting in series the electrical resistances, as shown in Fig. 5. This thermal network is valid for $e \ll L$.

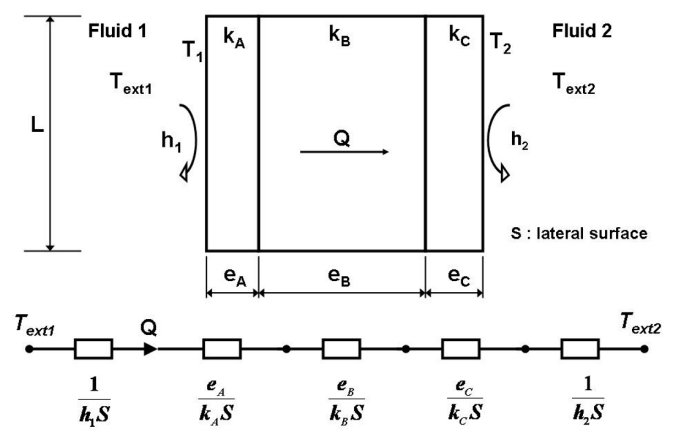

Fig. 5. Multilayer wall and its equivalent electric circuit

\section{B. Thermal Network for a Peltier Module}

Peltier modules are active elements of the submerged freeze microgripper. As described above, they are controlled by electrical current. The cooling capacity $Q_{c}$ of a Peltier module is related to its Peltier coefficient $\alpha$, its electrical resistance $R$, its thermal conductivity coefficient $k_{P}$, the electrical current supplied $i$, and the temperature of its cold and hot face, $T_{c}$ and $T_{h}$ respectively. The heat flow $Q_{h}$ of the hot face can be obtained by symmetries. They can be written as:

$$
\begin{aligned}
Q_{c} & =-\alpha T_{c} i+R i^{2} / 2+k_{P}\left(T_{h}-T_{c}\right) \\
Q_{h} & =\alpha T_{h} i+R i^{2} / 2-k_{P}\left(T_{h}-T_{c}\right)
\end{aligned}
$$

The thermal network for a Peltier module is described by [13]. The steady state thermal network based on (3) and (4) is described in Fig. 6. Peltier effect is represented by current source $P_{S}=\alpha T_{c} i$; Joule effect is modelled by current sources $P_{J / 2}=R i^{2} / 2$; and the conductivity coefficient is analogous to the thermal resistance term, $R_{t h}=1 / k_{P}$.

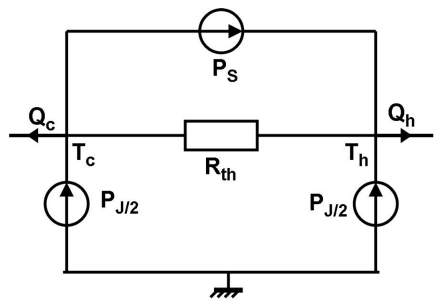

Fig. 6. Thermal network for a Peltier element [13]

In order to validate the performance of the MiniPeltier, we compared its net cooling, i.e. $T_{h}-T_{c}$, obtained with the electrical model with experimental measurements. According to Fig. 6, net cooling is written as:

$$
T_{h}-T_{c}=R_{t h}\left(Q_{c}+P_{S}-P_{J / 2}\right) .
$$

All the parameters are provided by the MiniPeltier's handbook. Temperatures have been measured using a microthermocouple on each face (cold face and hot face) of the MiniPeltier.

Fig. 7 shows electrical modelled and experimental results and is composed of successive steady-state situations when the electrical current is ranged from 0 to 1.1 A. Despite the fact that the electrical model does not take into account other sources of heat loss, like connections or junctions, which impact increases with the electrical current, the model seems statisfactory.

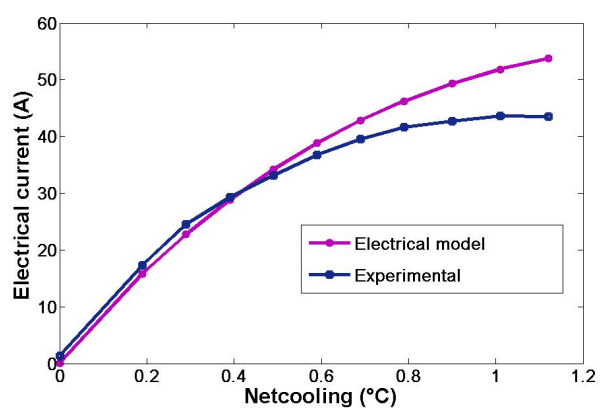

Fig. 7. Comparison between the electrical modelled net cooling and the experimental net cooling

\section{Thermal Network for a 3D Thermal Problem}

In addition to the Peltier modules, the submerged freeze gripper consists also on passive elements, like the end-effector and the heat sinks. Then the global thermal behaviour of the gripper involves a combination of conduction and convection effects. As shown in Fig. 8, the heat flux $Q$ takes place between the two lateral 
temperatures $T_{1}$ and $T_{2}$, and heat convection $Q_{h}$ occurs from all other surfaces in contact with the external fluid whose temperature is $T_{\text {ext }}$.

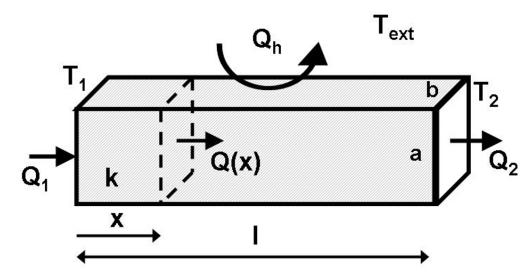

Fig. 8. Thermal schema of passive elements

This configuration is quite different from the typical example illustrated in Fig. 5. In order to obtain the equivalent electrical model for these passive elements, we establish the relationship between the heat flux, and the convection and conduction terms as:

$$
Q_{1}=\frac{T_{1}-T_{e x t}}{R_{v}}+\frac{T_{1}-T_{2}}{R_{c}},
$$

where $Q_{1}$ is the heat flux on the left lateral surface, $R_{c}$ is the thermal conduction resistance, and $R_{v}$ is the thermal convection resistance.

The heat flux in the right lateral surface $Q_{2}$ can be obtained by symmetry of the heat flux $Q_{1}$ in (6):

$$
Q_{2}=\frac{T_{2}-T_{e x t}}{R_{v}}+\frac{T_{2}-T_{1}}{R_{c}} .
$$

Equations (6) and (7) implies the thermal network of the Fig. 9.

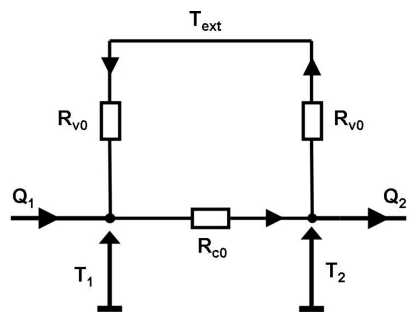

Fig. 9. Equivalent electric model for passive elements

The calculation of the thermal resistances required the determination of the heat flux $Q(x)$. The heat balance for the volume given in Fig. 8 gives:

$$
Q(x)=Q(x+d x)+d Q_{h} .
$$

The conduction through the element is:

$$
Q(x)=-k S \frac{d T}{d x},
$$

and the convection to the environment is:

$$
d Q_{h}=-h P d x\left(T(x)-T_{e x t}\right),
$$

where $P=2 a+2 b$ is the perimeter, and $S=a b$ is the section. Parameters a, b and l, defined in Fig. 8, corresponds on height, width and length respectively.

Considering (8), (9), and (10), the temperature $T(x)$ of the element verifies:

$$
\begin{aligned}
L^{2} \frac{d^{2} T(x)}{d x^{2}}-T(x) & =-T_{e x t}, \\
\text { where: } L & =\sqrt{k S / h P} .
\end{aligned}
$$

After solving (11) to obtain the temperature $T(x)$, and introducing the solution in (9), the heat flux expression can be determined as:

$$
\begin{aligned}
Q(x)= & -\frac{k S}{L}\left[\left(T_{1}-T_{e x t}\right)(\sinh (x / L)\right. \\
& \left.+\frac{\cosh (x / L)}{\sinh (l / L)}(1-\cosh (l / L))\right) \\
& \left.+\left(T_{2}-T_{1}\right) \frac{\cosh (x / L)}{\sinh (l / L)}\right] .
\end{aligned}
$$

For $x=0$, the heat flux $Q(x)$ becomes $Q_{1}$, given by:

$$
Q_{1}=\frac{-k S\left[\left(T_{1}-T_{e x t}\right)(1-\cosh (l / L))+\left(T_{2}-T_{1}\right)\right]}{L \sinh (l / L)} .
$$

Associating (6) and (14), the thermal resistances $R_{c}$ and $R_{v}$ can be written as:

$$
\begin{aligned}
R_{c} & =\frac{L \sinh (l / L)}{k S}, \\
R_{v} & =\frac{L \sinh (l / L)}{k S(\cosh (l / L)-1)} .
\end{aligned}
$$

Both expressions can be also written as:

$$
\begin{aligned}
& R_{c}=\frac{l}{k S}\left(\frac{\sinh (l / L)}{(l / L)}\right) \approx \frac{l}{k S}\left(1+\frac{(l / L)^{2}}{6}\right), \\
& R_{v}=\frac{2}{P l h}\left(\frac{(l / L) \sinh (l / L)}{2(\cosh (l / L)-1)}\right) \approx \frac{2}{P l h}\left(1+\frac{(l / L)^{2}}{6}\right) .
\end{aligned}
$$

Consequently, for small ratio $l / L$ verifying the inequality:

$$
\delta=\frac{(l / L)^{2}}{6} \ll 1,
$$

the convection and conduction resistances can be simplified as:

$$
\begin{aligned}
& R_{c 0}=l / k S, \\
& R_{v 0}=2 / P l h .
\end{aligned}
$$


To validate the thermal network, the left lateral heat $Q_{1}$ and the right lateral heat $Q_{2}$ for different geometries have been calculated in water and air, using three different models: the analytical model applying (13); a finite element (FEM) calculation simulated with COMSOL Multiphysics 3.2; and the thermal network in Fig. 9. The experimental validation of the whole system will be presented in a next work.

The comparison of the values for several cases is presented in Table III. Let us note that the criterion $\delta$ defined in (19) represents the relevance of the thermal network. The first case for each medium (water and air) corresponds to the MicroPelt's heat sink geometry.

TABLE III

Comparison of Heat Flux $Q_{1}$ and $Q_{2}$

\begin{tabular}{|l|c|l|c|c|}
\hline $\begin{array}{l}\text { Geometry } \\
(\mathrm{mm})\end{array}$ & $\delta(\mathbf{1 9})$ & Model & $\begin{array}{c}Q_{1} \\
(\mathrm{~W})\end{array}$ & $\begin{array}{c}Q_{2} \\
(\mathrm{~W})\end{array}$ \\
\hline \multicolumn{4}{|c|}{ Comparison in air } \\
\hline$a=2$ & $0.040<1$ & Analytical & -0.023 & 1.025 \\
$b=7$ & & FEM & -0.025 & 1.029 \\
$l=22$ & & Electrical & -0.014 & 1.056 \\
\hline$a=50$ & $0.001<1$ & Analytical & 560 & 598 \\
$b=300$ & & FEM & 559 & 600 \\
$l=20$ & & Electrical & 561 & 599 \\
\hline \multicolumn{5}{|c|}{ Comparison in water } \\
\hline$a=2$ & $0.403<1$ & Analytical & -3.89 & 5.07 \\
$b=7$ & & FEM & -3.90 & 5.09 \\
$l=22$ & & Electrical & -4.56 & 6.13 \\
\hline$a=50$ & $0.012<1$ & Analytical & 395 & 770 \\
$b=300$ & & FEM & 382 & 779 \\
$l=20$ & & Electrical & 401 & 779 \\
\hline
\end{tabular}

The results obtained comparing either analytical or FEM calculation with the static thermal network allow us to validate it.

A whole system thermal network could then estimate properly heat processes, analyse clearly the parameters and finally is of great help for the design and optimization of the submerged freeze microgripper. Further works will focus on dynamic modelling, characterization of the parameters involved in the whole thermal network and its experimental validation.

\section{CONCLUSIONS AND FUTURE WORKS}

An innovative handling strategy to manipulate microobjects whose typical size is under $100 \mu \mathrm{m}$ has been developed and successfully tested. A comparative analysis of the interaction of dry and liquid media on surface forces, contact forces, and hydrodynamic forces have shown the interest of submerged artificial micro-objects manipulations. This approach permits new micromanipulation strategies adapted to the aqueous medium. A freeze gripper for such medium is proposed: an ice droplet is generated at the active part of the end-effector to pick up the object; to release it, the ice is thawed without having influence of the capillary force. During first experimentations the advantages of the liquid medium were exhibited: adhesion perturbations reduce considerably. The submerged freeze gripper is thus a promising approach to execute reliable and flexible micromanipulation and micro-assembly tasks.

To control and optimize the heat exchanges involved in the handling process and its cycle time, an equivalent electrical model is proposed. The thermal network for a Peltier module has been presented and compared to the MiniPeltier's experimental results. A new thermal network for a 3D heat sink has been also developed and compared to an analytical model and a FEM model. In all cases, the equivalent electrical model in steady state seems sufficiently accurate.

Dynamic thermal modelling is currently under development. This model will allow us in the next step to control the temperature and thickness of the ice microdrop.

\section{REFERENCES}

[1] H. Van Brussel et al. Assembly of microsystems. In Annals of the CIRP, volume 49, pages 451-472, 2000.

[2] N. Dechev, W. L. Cleghorn, and J. K. Mills. Microassembly of 3-d microstructures using a compliant, passive microgripper. Journal of Microelectromechanical Systems, 13(12):176-189, April 2004.

[3] T. Udeshi and K. Tsui. Assembly sequence planning for automated micro assembly. In Int. Symp. on Assembly and Task Planning, pages 98-105, 2005.

[4] M. Savia, Q. Zhou, and H. N. Koivo. Simulating adhesion forces between arbitrarily shaped objects in micro/nanohandling operations. In Proc. of the IEEE/RSJ IROS, pages 1772-1727, Sendai, Japan, October 2004.

[5] Q. Zhou, B. Chang, and H. N. Koivo. Ambient environment effects in micro/nano handling. In Proc. of the $I W M F$, pages 146-151, Shangai, China, October 2004.

[6] J. Israelachvili. Intermolecular and surfaces forces. Academic Press, 1991.

[7] Z. Xu and R. H. Yoon. The role of hydrophobic interactions in coagulation. Journal of Colloid Interface Science, 44(132):532-541, 1989.

[8] Z. Xu and R. H. Yoon. A study of hydrophobic coagulation. Journal of Colloid Interface Science, 45(134):427-434, 1990.

[9] M. Gauthier, B. López-Walle, and C. Clévy. Comparison between micro-objects manipulations in dry and liquid mediums. In Proc. of the 6th CIRA, Espoo, Finland, June 2005.

[10] M. Gauthier et al. Forces analyses for micromanipulations in dry and liquid media. Journal of Micromechatronics, 3(34):389-413, September 2006.

[11] J. Liu, Y.-X. Zhou, and T.-H. Yu. Freeze tweezer to manipulate mini/micro objects. JMM, 14(2):269-276, February 2004.

[12] S. Droz et al. New generation of grippers for the manipulation of miniaturized components. In Proc. of Mechatronics, pages 572-575, Besançon, France, October 2001.

[13] G. Seliger, J. Stephan, and S. Lange. Hydroadhesive gripping by using peltier effect. In Proc. of IMECE, pages 3-8, Florida, USA, November 2000.

[14] B. López-Walle, M. Gauthier, and N. Chaillet. Submerged freeze gripper to manipulate micro-objects. In Proc. IEEE/RSJ IROS, Beijing, China, October 2006.

[15] J. P. Holman. Heat transfer. Mac Graw Hill, 1990. 